\title{
Quest for the best-A move to Anatomical Endoscopic Enucleation of the Prostate
}

The history of surgical enucleation for the treatment of lower urinary tract symptoms caused by benign prostatic enlargement dates back more than 100 years (Freyer, 1919). Open prostatectomy (OP) is an invasive procedure associated with high transfusion rates, long catheterisation time, and long hospital stay in spite of its capability to achieve complete removal of prostatic adenoma. As a result, the popularity of OP has declined after the advent of transurethral resection of the prostate (TURP). It is not surprising that TURP has been considered the standard surgical therapy to treat benign prostatic hyperplasia (BPH) for decades in view of its favourable safety profile and minimally invasive nature. Despite improvements in equipment and techniques over the years, morbidity and retreatment rates after TURP are still of concern, particularly in patients with a large prostate (Rassweiler, Teber, Kuntz, \& Hofmann, 2006). The ongoing needs to perfect BPH-related surgery lead to advances in new technologies and refinement of current options. Among all the surgical options, anatomical endoscopic enucleation of the prostate (AEEP) is the most promising one.

The concept of surgical enucleation by an endoscopic approach was first described by Hiraoka in 1983 with the use of a monopolar system (Hiraoka, 1983). However, AEEP did not attract much attention from the urological world until holmium laser enucleation of the prostate (HoLEP) was introduced by Fraundorfer and Gilling in 1998 (Fraundorfer \& Gilling, 1998). Since then, AEEP has undergone growing popularity due to its achievement of maximal adenoma removal with significantly less morbidity. HoLEP remains the most well-studied procedure in AEEP and has demonstrated its superior outcome efficacy, durability and safety (Gilling et al., 2012). Effort and data from researchers worldwide brought the acronymEndoscopic Enucleation of the Prostate (EEP)-to the European Association of Urology Guidelines in 2016 (Gravas et al., 2016). Nowadays, AEEP continues to evolve rapidly and is not only limited to HoLEP. Various energy sources and a wide spectrum of operative techniques have been adopted by different surgeons with success. Nonetheless, anatomical enucleation is the core principle, which is shared by all the endourologists despite the variations.

While AEEP continues to flourish and becomes available in an increasing number of centres globally, numerous questions remain to be answered. In our quest for generating a wealth of scientific information, the Guest Editors have invited a panel of experts in the field to present the current best evidence on AEEP. The Special
Issue involves a comprehensive list of chapters and offers a con-
cise overview of AEEP in a systematic approach. Following the in-
troduction from the historical and anatomical perspective (Reddy,
Utley, \& Gilling, 2020; Oh \& Shitara, 2020), different techniques
of AEEP by different energy source are illustrated (Ryang, Ly, Tran,
Oh, \& Cho, 2020; de Figueiredo, Cracco, de Marins, \& Scoffone,
$2020 ;$ Herrmann \& Wolters, 2020; Rijo \& Misrai, 2020). The surgical
outcomes, with emphasis on postoperative continence and sexual
functions, and complications of AEEP are summarised in the fol-
lowing chapters (Chen, Chung, Chu, Chen, \& Ho, 2020; Lee, Cho,
Juan, \& Teoh, 2020; Cheng, Li, \& Yu, 2020; Wei, Ke, Xu, \& Xue,
2020 ) before a debate on AEEP as the next gold standard treatment
for benign prostatic obstruction (Aho, Armitage, \& Kastner, 2020;
Wroclawski, Teles, \& Carneiro, 2020). Finally, this Special Issue is
rounded up by a discussion on surgical training (Teoh et al., 2020)
and a survey on AEEP from urologists worldwide (Gudaru et al.,
2020 ).
Since the awakening of the procedure 20 years ago, we witnessed
the enormous development in every aspect of AEEP. Therefore, we
believe that it is high time to summarise the advancement of the
technique so far. This Special Issue serves as a firm foundation by
compiling the achievements of researchers around the world and
shedding light on generalisation of the technique of AEEP.

Issue involves a comprehensive list of chapters and offers a con-
cise overview of AEEP in a systematic approach. Following the in-
troduction from the historical and anatomical perspective (Reddy,
Utley, \& Gilling, 2020; Oh \& Shitara, 2020), different techniques
of AEEP by different energy source are illustrated (Ryang, Ly, Tran,
Oh, \& Cho, 2020; de Figueiredo, Cracco, de Marins, \& Scoffone,
$2020 ;$ Herrmann \& Wolters, 2020; Rijo \& Misrai, 2020). The surgical
outcomes, with emphasis on postoperative continence and sexual
functions, and complications of AEEP are summarised in the fol-
lowing chapters (Chen, Chung, Chu, Chen, \& Ho, 2020; Lee, Cho,
Juan, \& Teoh, 2020; Cheng, Li, \& Yu, 2020; Wei, Ke, Xu, \& Xue,
2020 ) before a debate on AEEP as the next gold standard treatment
for benign prostatic obstruction (Aho, Armitage, \& Kastner, 2020;
Wroclawski, Teles, \& Carneiro, 2020). Finally, this Special Issue is
rounded up by a discussion on surgical training (Teoh et al., 2020)
and a survey on AEEP from urologists worldwide (Gudaru et al.,
2020 ).
Since the awakening of the procedure 20 years ago, we witnessed
the enormous development in every aspect of AEEP. Therefore, we
believe that it is high time to summarise the advancement of the
technique so far. This Special Issue serves as a firm foundation by
compiling the achievements of researchers around the world and
shedding light on generalisation of the technique of AEEP.

Issue involves a comprehensive list of chapters and offers a con-
cise overview of AEEP in a systematic approach. Following the in-
troduction from the historical and anatomical perspective (Reddy,
Utley, \& Gilling, 2020; Oh \& Shitara, 2020), different techniques
of AEEP by different energy source are illustrated (Ryang, Ly, Tran,
Oh, \& Cho, 2020; de Figueiredo, Cracco, de Marins, \& Scoffone,
$2020 ;$ Herrmann \& Wolters, 2020; Rijo \& Misrai, 2020). The surgical
outcomes, with emphasis on postoperative continence and sexual
functions, and complications of AEEP are summarised in the fol-
lowing chapters (Chen, Chung, Chu, Chen, \& Ho, 2020; Lee, Cho,
Juan, \& Teoh, 2020; Cheng, Li, \& Yu, 2020; Wei, Ke, Xu, \& Xue,
2020 ) before a debate on AEEP as the next gold standard treatment
for benign prostatic obstruction (Aho, Armitage, \& Kastner, 2020;
Wroclawski, Teles, \& Carneiro, 2020). Finally, this Special Issue is
rounded up by a discussion on surgical training (Teoh et al., 2020)
and a survey on AEEP from urologists worldwide (Gudaru et al.,
2020 ).
Since the awakening of the procedure 20 years ago, we witnessed
the enormous development in every aspect of AEEP. Therefore, we
believe that it is high time to summarise the advancement of the
technique so far. This Special Issue serves as a firm foundation by
compiling the achievements of researchers around the world and
shedding light on generalisation of the technique of AEEP.

Issue involves a comprehensive list of chapters and offers a con-
cise overview of AEEP in a systematic approach. Following the in-
troduction from the historical and anatomical perspective (Reddy,
Utley, \& Gilling, 2020; Oh \& Shitara, 2020), different techniques
of AEEP by different energy source are illustrated (Ryang, Ly, Tran,
Oh, \& Cho, 2020; de Figueiredo, Cracco, de Marins, \& Scoffone,
2020; Herrmann \& Wolters, 2020; Rijo \& Misrai, 2020). The surgical
outcomes, with emphasis on postoperative continence and sexual
functions, and complications of AEEP are summarised in the fol-
lowing chapters (Chen, Chung, Chu, Chen, \& Ho, 2020; Lee, Cho,
Juan, \& Teoh, 2020; Cheng, Li, \& Yu, 2020; Wei, Ke, Xu, \& Xue,
2020 ) before a debate on AEEP as the next gold standard treatment
for benign prostatic obstruction (Aho, Armitage, \& Kastner, 2020;
Wroclawski, Teles, \& Carneiro, 2020). Finally, this Special Issue is
rounded up by a discussion on surgical training (Teoh et al., 2020)
and a survey on AEEP from urologists worldwide (Gudaru et al.,
2020 ).
Since the awakening of the procedure 20 years ago, we witnessed
the enormous development in every aspect of AEEP. Therefore, we
believe that it is high time to summarise the advancement of the
technique so far. This Special Issue serves as a firm foundation by
compiling the achievements of researchers around the world and
shedding light on generalisation of the technique of AEEP.

Issue involves a comprehensive list of chapters and offers a con-
cise overview of AEEP in a systematic approach. Following the in-
troduction from the historical and anatomical perspective (Reddy,
Utley, \& Gilling, 2020; Oh \& Shitara, 2020), different techniques
of AEEP by different energy source are illustrated (Ryang, Ly, Tran,
Oh, \& Cho, 2020; de Figueiredo, Cracco, de Marins, \& Scoffone,
2020; Herrmann \& Wolters, 2020; Rijo \& Misrai, 2020). The surgical
outcomes, with emphasis on postoperative continence and sexual
functions, and complications of AEEP are summarised in the fol-
lowing chapters (Chen, Chung, Chu, Chen, \& Ho, 2020; Lee, Cho,
Juan, \& Teoh, 2020; Cheng, Li, \& Yu, 2020; Wei, Ke, Xu, \& Xue,
2020 ) before a debate on AEEP as the next gold standard treatment
for benign prostatic obstruction (Aho, Armitage, \& Kastner, 2020;
Wroclawski, Teles, \& Carneiro, 2020). Finally, this Special Issue is
rounded up by a discussion on surgical training (Teoh et al., 2020)
and a survey on AEEP from urologists worldwide (Gudaru et al.,
2020 ).
Since the awakening of the procedure 20 years ago, we witnessed
the enormous development in every aspect of AEEP. Therefore, we
believe that it is high time to summarise the advancement of the
technique so far. This Special Issue serves as a firm foundation by
compiling the achievements of researchers around the world and
shedding light on generalisation of the technique of AEEP.

Issue involves a comprehensive list of chapters and offers a con-
cise overview of AEEP in a systematic approach. Following the in-
troduction from the historical and anatomical perspective (Reddy,
Utley, \& Gilling, 2020; Oh \& Shitara, 2020), different techniques
of AEEP by different energy source are illustrated (Ryang, Ly, Tran,
Oh, \& Cho, 2020; de Figueiredo, Cracco, de Marins, \& Scoffone,
2020; Herrmann \& Wolters, 2020; Rijo \& Misrai, 2020). The surgical
outcomes, with emphasis on postoperative continence and sexual
functions, and complications of AEEP are summarised in the fol-
lowing chapters (Chen, Chung, Chu, Chen, \& Ho, 2020; Lee, Cho,
Juan, \& Teoh, 2020; Cheng, Li, \& Yu, 2020; Wei, Ke, Xu, \& Xue,
2020 ) before a debate on AEEP as the next gold standard treatment
for benign prostatic obstruction (Aho, Armitage, \& Kastner, 2020;
Wroclawski, Teles, \& Carneiro, 2020). Finally, this Special Issue is
rounded up by a discussion on surgical training (Teoh et al., 2020)
and a survey on AEEP from urologists worldwide (Gudaru et al.,
2020 ).
Since the awakening of the procedure 20 years ago, we witnessed
the enormous development in every aspect of AEEP. Therefore, we
believe that it is high time to summarise the advancement of the
technique so far. This Special Issue serves as a firm foundation by
compiling the achievements of researchers around the world and
shedding light on generalisation of the technique of AEEP.

Issue involves a comprehensive list of chapters and offers a con-
cise overview of AEEP in a systematic approach. Following the in-
troduction from the historical and anatomical perspective (Reddy,
Utley, \& Gilling, 2020; Oh \& Shitara, 2020), different techniques
of AEEP by different energy source are illustrated (Ryang, Ly, Tran,
Oh, \& Cho, 2020; de Figueiredo, Cracco, de Marins, \& Scoffone,
$2020 ;$ Herrmann \& Wolters, 2020; Rijo \& Misrai, 2020). The surgical
outcomes, with emphasis on postoperative continence and sexual
functions, and complications of AEEP are summarised in the fol-
lowing chapters (Chen, Chung, Chu, Chen, \& Ho, 2020; Lee, Cho,
Juan, \& Teoh, 2020; Cheng, Li, \& Yu, 2020; Wei, Ke, Xu, \& Xue,
2020 ) before a debate on AEEP as the next gold standard treatment
for benign prostatic obstruction (Aho, Armitage, \& Kastner, 2020;
Wroclawski, Teles, \& Carneiro, 2020). Finally, this Special Issue is
rounded up by a discussion on surgical training (Teoh et al., 2020)
and a survey on AEEP from urologists worldwide (Gudaru et al.,
2020 ).
Since the awakening of the procedure 20 years ago, we witnessed
the enormous development in every aspect of AEEP. Therefore, we
believe that it is high time to summarise the advancement of the
technique so far. This Special Issue serves as a firm foundation by
compiling the achievements of researchers around the world and
shedding light on generalisation of the technique of AEEP.

Issue involves a comprehensive list of chapters and offers a con-
cise overview of AEEP in a systematic approach. Following the in-
troduction from the historical and anatomical perspective (Reddy,
Utley, \& Gilling, 2020; Oh \& Shitara, 2020), different techniques
of AEEP by different energy source are illustrated (Ryang, Ly, Tran,
Oh, \& Cho, 2020; de Figueiredo, Cracco, de Marins, \& Scoffone,
2020; Herrmann \& Wolters, 2020; Rijo \& Misrai, 2020). The surgical
outcomes, with emphasis on postoperative continence and sexual
functions, and complications of AEEP are summarised in the fol-
lowing chapters (Chen, Chung, Chu, Chen, \& Ho, 2020; Lee, Cho,
Juan, \& Teoh, 2020; Cheng, Li, \& Yu, 2020; Wei, Ke, Xu, \& Xue,
2020 ) before a debate on AEEP as the next gold standard treatment
for benign prostatic obstruction (Aho, Armitage, \& Kastner, 2020;
Wroclawski, Teles, \& Carneiro, 2020). Finally, this Special Issue is
rounded up by a discussion on surgical training (Teoh et al., 2020)
and a survey on AEEP from urologists worldwide (Gudaru et al.,
2020 ).
Since the awakening of the procedure 20 years ago, we witnessed
the enormous development in every aspect of AEEP. Therefore, we
believe that it is high time to summarise the advancement of the
technique so far. This Special Issue serves as a firm foundation by
compiling the achievements of researchers around the world and
shedding light on generalisation of the technique of AEEP.

Issue involves a comprehensive list of chapters and offers a con-
cise overview of AEEP in a systematic approach. Following the in-
troduction from the historical and anatomical perspective (Reddy,
Utley, \& Gilling, 2020; Oh \& Shitara, 2020), different techniques
of AEEP by different energy source are illustrated (Ryang, Ly, Tran,
Oh, \& Cho, 2020; de Figueiredo, Cracco, de Marins, \& Scoffone,
$2020 ;$ Herrmann \& Wolters, 2020; Rijo \& Misrai, 2020). The surgical
outcomes, with emphasis on postoperative continence and sexual
functions, and complications of AEEP are summarised in the fol-
lowing chapters (Chen, Chung, Chu, Chen, \& Ho, 2020; Lee, Cho,
Juan, \& Teoh, 2020; Cheng, Li, \& Yu, 2020; Wei, Ke, Xu, \& Xue,
2020 ) before a debate on AEEP as the next gold standard treatment
for benign prostatic obstruction (Aho, Armitage, \& Kastner, 2020;
Wroclawski, Teles, \& Carneiro, 2020). Finally, this Special Issue is
rounded up by a discussion on surgical training (Teoh et al., 2020)
and a survey on AEEP from urologists worldwide (Gudaru et al.,
2020 ).
Since the awakening of the procedure 20 years ago, we witnessed
the enormous development in every aspect of AEEP. Therefore, we
believe that it is high time to summarise the advancement of the
technique so far. This Special Issue serves as a firm foundation by
compiling the achievements of researchers around the world and
shedding light on generalisation of the technique of AEEP.

Issue involves a comprehensive list of chapters and offers a con-
cise overview of AEEP in a systematic approach. Following the in-
troduction from the historical and anatomical perspective (Reddy,
Utley, \& Gilling, 2020; Oh \& Shitara, 2020), different techniques
of AEEP by different energy source are illustrated (Ryang, Ly, Tran,
Oh, \& Cho, 2020; de Figueiredo, Cracco, de Marins, \& Scoffone,
2020; Herrmann \& Wolters, 2020; Rijo \& Misrai, 2020). The surgical
outcomes, with emphasis on postoperative continence and sexual
functions, and complications of AEEP are summarised in the fol-
lowing chapters (Chen, Chung, Chu, Chen, \& Ho, 2020; Lee, Cho,
Juan, \& Teoh, 2020; Cheng, Li, \& Yu, 2020; Wei, Ke, Xu, \& Xue,
2020 ) before a debate on AEEP as the next gold standard treatment
for benign prostatic obstruction (Aho, Armitage, \& Kastner, 2020;
Wroclawski, Teles, \& Carneiro, 2020). Finally, this Special Issue is
rounded up by a discussion on surgical training (Teoh et al., 2020)
and a survey on AEEP from urologists worldwide (Gudaru et al.,
2020 ).
Since the awakening of the procedure 20 years ago, we witnessed
the enormous development in every aspect of AEEP. Therefore, we
believe that it is high time to summarise the advancement of the
technique so far. This Special Issue serves as a firm foundation by
compiling the achievements of researchers around the world and
shedding light on generalisation of the technique of AEEP.

Issue involves a comprehensive list of chapters and offers a con-
cise overview of AEEP in a systematic approach. Following the in-
troduction from the historical and anatomical perspective (Reddy,
Utley, \& Gilling, 2020; Oh \& Shitara, 2020), different techniques
of AEEP by different energy source are illustrated (Ryang, Ly, Tran,
Oh, \& Cho, 2020; de Figueiredo, Cracco, de Marins, \& Scoffone,
2020; Herrmann \& Wolters, 2020; Rijo \& Misrai, 2020). The surgical
outcomes, with emphasis on postoperative continence and sexual
functions, and complications of AEEP are summarised in the fol-
lowing chapters (Chen, Chung, Chu, Chen, \& Ho, 2020; Lee, Cho,
Juan, \& Teoh, 2020; Cheng, Li, \& Yu, 2020; Wei, Ke, Xu, \& Xue,
2020 ) before a debate on AEEP as the next gold standard treatment
for benign prostatic obstruction (Aho, Armitage, \& Kastner, 2020;
Wroclawski, Teles, \& Carneiro, 2020). Finally, this Special Issue is
rounded up by a discussion on surgical training (Teoh et al., 2020)
and a survey on AEEP from urologists worldwide (Gudaru et al.,
2020 ).
Since the awakening of the procedure 20 years ago, we witnessed
the enormous development in every aspect of AEEP. Therefore, we
believe that it is high time to summarise the advancement of the
technique so far. This Special Issue serves as a firm foundation by
compiling the achievements of researchers around the world and
shedding light on generalisation of the technique of AEEP.

Issue involves a comprehensive list of chapters and offers a con-
cise overview of AEEP in a systematic approach. Following the in-
troduction from the historical and anatomical perspective (Reddy,
Utley, \& Gilling, 2020; Oh \& Shitara, 2020), different techniques
of AEEP by different energy source are illustrated (Ryang, Ly, Tran,
Oh, \& Cho, 2020; de Figueiredo, Cracco, de Marins, \& Scoffone,
2020; Herrmann \& Wolters, 2020; Rijo \& Misrai, 2020). The surgical
outcomes, with emphasis on postoperative continence and sexual
functions, and complications of AEEP are summarised in the fol-
lowing chapters (Chen, Chung, Chu, Chen, \& Ho, 2020; Lee, Cho,
Juan, \& Teoh, 2020; Cheng, Li, \& Yu, 2020; Wei, Ke, Xu, \& Xue,
2020 ) before a debate on AEEP as the next gold standard treatment
for benign prostatic obstruction (Aho, Armitage, \& Kastner, 2020;
Wroclawski, Teles, \& Carneiro, 2020). Finally, this Special Issue is
rounded up by a discussion on surgical training (Teoh et al., 2020)
and a survey on AEEP from urologists worldwide (Gudaru et al.,
2020 ).
Since the awakening of the procedure 20 years ago, we witnessed
the enormous development in every aspect of AEEP. Therefore, we
believe that it is high time to summarise the advancement of the
technique so far. This Special Issue serves as a firm foundation by
compiling the achievements of researchers around the world and
shedding light on generalisation of the technique of AEEP.

Issue involves a comprehensive list of chapters and offers a con-
cise overview of AEEP in a systematic approach. Following the in-
troduction from the historical and anatomical perspective (Reddy,
Utley, \& Gilling, 2020; Oh \& Shitara, 2020), different techniques
of AEEP by different energy source are illustrated (Ryang, Ly, Tran,
Oh, \& Cho, 2020; de Figueiredo, Cracco, de Marins, \& Scoffone,
2020; Herrmann \& Wolters, 2020; Rijo \& Misrai, 2020). The surgical
outcomes, with emphasis on postoperative continence and sexual
functions, and complications of AEEP are summarised in the fol-
lowing chapters (Chen, Chung, Chu, Chen, \& Ho, 2020; Lee, Cho,
Juan, \& Teoh, 2020; Cheng, Li, \& Yu, 2020; Wei, Ke, Xu, \& Xue,
2020 ) before a debate on AEEP as the next gold standard treatment
for benign prostatic obstruction (Aho, Armitage, \& Kastner, 2020;
Wroclawski, Teles, \& Carneiro, 2020). Finally, this Special Issue is
rounded up by a discussion on surgical training (Teoh et al., 2020)
and a survey on AEEP from urologists worldwide (Gudaru et al.,
2020 ).
Since the awakening of the procedure 20 years ago, we witnessed
the enormous development in every aspect of AEEP. Therefore, we
believe that it is high time to summarise the advancement of the
technique so far. This Special Issue serves as a firm foundation by
compiling the achievements of researchers around the world and
shedding light on generalisation of the technique of AEEP.

Issue involves a comprehensive list of chapters and offers a con-
cise overview of AEEP in a systematic approach. Following the in-
troduction from the historical and anatomical perspective (Reddy,
Utley, \& Gilling, 2020; Oh \& Shitara, 2020), different techniques
of AEEP by different energy source are illustrated (Ryang, Ly, Tran,
Oh, \& Cho, 2020; de Figueiredo, Cracco, de Marins, \& Scoffone,
$2020 ;$ Herrmann \& Wolters, 2020; Rijo \& Misrai, 2020). The surgical
outcomes, with emphasis on postoperative continence and sexual
functions, and complications of AEEP are summarised in the fol-
lowing chapters (Chen, Chung, Chu, Chen, \& Ho, 2020; Lee, Cho,
Juan, \& Teoh, 2020; Cheng, Li, \& Yu, 2020; Wei, Ke, Xu, \& Xue,
2020 ) before a debate on AEEP as the next gold standard treatment
for benign prostatic obstruction (Aho, Armitage, \& Kastner, 2020;
Wroclawski, Teles, \& Carneiro, 2020). Finally, this Special Issue is
rounded up by a discussion on surgical training (Teoh et al., 2020)
and a survey on AEEP from urologists worldwide (Gudaru et al.,
2020 ).
Since the awakening of the procedure 20 years ago, we witnessed
the enormous development in every aspect of AEEP. Therefore, we
believe that it is high time to summarise the advancement of the
technique so far. This Special Issue serves as a firm foundation by
compiling the achievements of researchers around the world and
shedding light on generalisation of the technique of AEEP.

Issue involves a comprehensive list of chapters and offers a con-
cise overview of AEEP in a systematic approach. Following the in-
troduction from the historical and anatomical perspective (Reddy,
Utley, \& Gilling, 2020; Oh \& Shitara, 2020), different techniques
of AEEP by different energy source are illustrated (Ryang, Ly, Tran,
Oh, \& Cho, 2020; de Figueiredo, Cracco, de Marins, \& Scoffone,
$2020 ;$ Herrmann \& Wolters, 2020; Rijo \& Misrai, 2020). The surgical
outcomes, with emphasis on postoperative continence and sexual
functions, and complications of AEEP are summarised in the fol-
lowing chapters (Chen, Chung, Chu, Chen, \& Ho, 2020; Lee, Cho,
Juan, \& Teoh, 2020; Cheng, Li, \& Yu, 2020; Wei, Ke, Xu, \& Xue,
2020 ) before a debate on AEEP as the next gold standard treatment
for benign prostatic obstruction (Aho, Armitage, \& Kastner, 2020;
Wroclawski, Teles, \& Carneiro, 2020). Finally, this Special Issue is
rounded up by a discussion on surgical training (Teoh et al., 2020)
and a survey on AEEP from urologists worldwide (Gudaru et al.,
2020 ).
Since the awakening of the procedure 20 years ago, we witnessed
the enormous development in every aspect of AEEP. Therefore, we
believe that it is high time to summarise the advancement of the
technique so far. This Special Issue serves as a firm foundation by
compiling the achievements of researchers around the world and
shedding light on generalisation of the technique of AEEP.

Issue involves a comprehensive list of chapters and offers a con-
cise overview of AEEP in a systematic approach. Following the in-
troduction from the historical and anatomical perspective (Reddy,
Utley, \& Gilling, 2020; Oh \& Shitara, 2020), different techniques
of AEEP by different energy source are illustrated (Ryang, Ly, Tran,
Oh, \& Cho, 2020; de Figueiredo, Cracco, de Marins, \& Scoffone,
2020; Herrmann \& Wolters, 2020; Rijo \& Misrai, 2020). The surgical
outcomes, with emphasis on postoperative continence and sexual
functions, and complications of AEEP are summarised in the fol-
lowing chapters (Chen, Chung, Chu, Chen, \& Ho, 2020; Lee, Cho,
Juan, \& Teoh, 2020; Cheng, Li, \& Yu, 2020; Wei, Ke, Xu, \& Xue,
2020 ) before a debate on AEEP as the next gold standard treatment
for benign prostatic obstruction (Aho, Armitage, \& Kastner, 2020;
Wroclawski, Teles, \& Carneiro, 2020). Finally, this Special Issue is
rounded up by a discussion on surgical training (Teoh et al., 2020)
and a survey on AEEP from urologists worldwide (Gudaru et al.,
2020 ).
Since the awakening of the procedure 20 years ago, we witnessed
the enormous development in every aspect of AEEP. Therefore, we
believe that it is high time to summarise the advancement of the
technique so far. This Special Issue serves as a firm foundation by
compiling the achievements of researchers around the world and
shedding light on generalisation of the technique of AEEP. 2020).

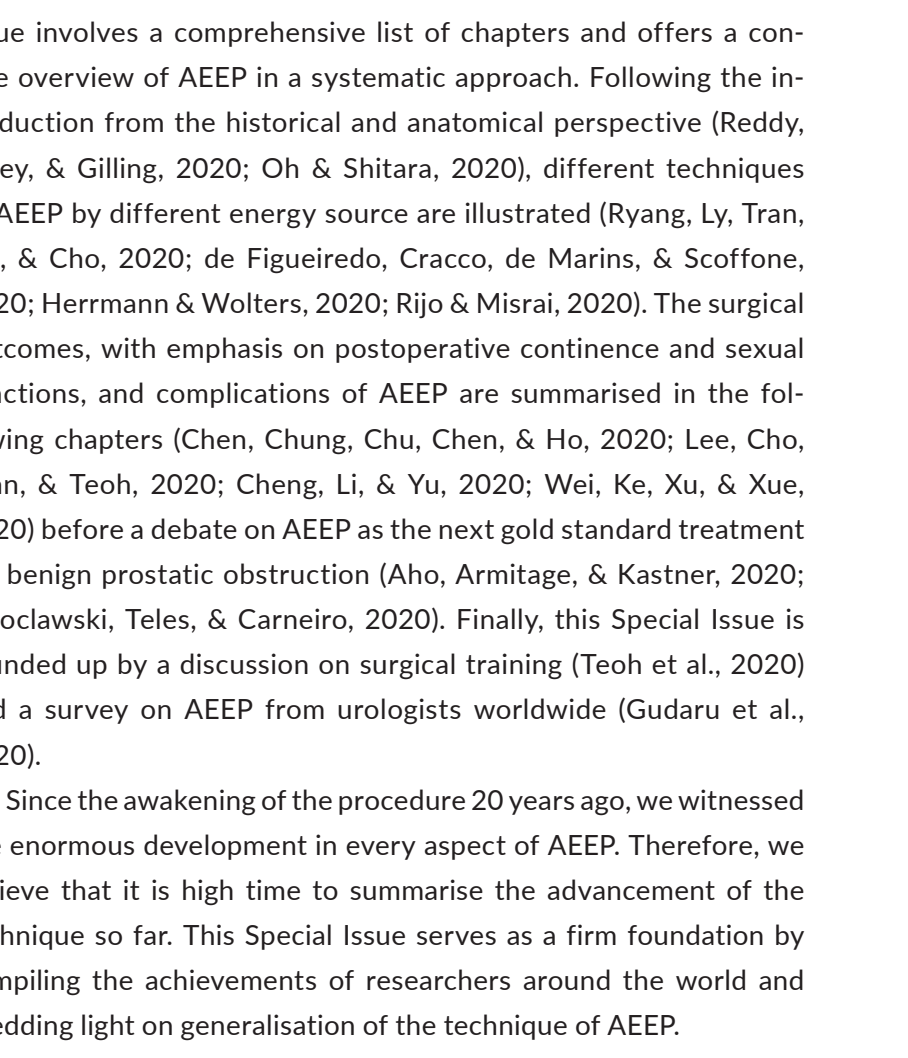

Issue involves a comprehensive list of chapters and offers a con-
cise overview of AEEP in a systematic approach. Following the in-
troduction from the historical and anatomical perspective (Reddy,
Utley, \& Gilling, 2020; Oh \& Shitara, 2020), different techniques
of AEEP by different energy source are illustrated (Ryang, Ly, Tran,
Oh, \& Cho, 2020; de Figueiredo, Cracco, de Marins, \& Scoffone,
2020; Herrmann \& Wolters, 2020; Rijo \& Misrai, 2020). The surgical
outcomes, with emphasis on postoperative continence and sexual
functions, and complications of AEEP are summarised in the fol-
lowing chapters (Chen, Chung, Chu, Chen, \& Ho, 2020; Lee, Cho,
Juan, \& Teoh, 2020; Cheng, Li, \& Yu, 2020; Wei, Ke, Xu, \& Xue,
2020 ) before a debate on AEEP as the next gold standard treatment
for benign prostatic obstruction (Aho, Armitage, \& Kastner, 2020;
Wroclawski, Teles, \& Carneiro, 2020). Finally, this Special Issue is
rounded up by a discussion on surgical training (Teoh et al., 2020)
and a survey on AEEP from urologists worldwide (Gudaru et al.,
2020 ).
Since the awakening of the procedure 20 years ago, we witnessed
the enormous development in every aspect of AEEP. Therefore, we
believe that it is high time to summarise the advancement of the
technique so far. This Special Issue serves as a firm foundation by
compiling the achievements of researchers around the world and
shedding light on generalisation of the technique of AEEP.

Issue involves a comprehensive list of chapters and offers a con-
cise overview of AEEP in a systematic approach. Following the in-
troduction from the historical and anatomical perspective (Reddy,
Utley, \& Gilling, 2020; Oh \& Shitara, 2020), different techniques
of AEEP by different energy source are illustrated (Ryang, Ly, Tran,
Oh, \& Cho, 2020; de Figueiredo, Cracco, de Marins, \& Scoffone,
2020; Herrmann \& Wolters, 2020; Rijo \& Misrai, 2020). The surgical
outcomes, with emphasis on postoperative continence and sexual
functions, and complications of AEEP are summarised in the fol-
lowing chapters (Chen, Chung, Chu, Chen, \& Ho, 2020; Lee, Cho,
Juan, \& Teoh, 2020; Cheng, Li, \& Yu, 2020; Wei, Ke, Xu, \& Xue,
2020 ) before a debate on AEEP as the next gold standard treatment
for benign prostatic obstruction (Aho, Armitage, \& Kastner, 2020;
Wroclawski, Teles, \& Carneiro, 2020). Finally, this Special Issue is
rounded up by a discussion on surgical training (Teoh et al., 2020)
and a survey on AEEP from urologists worldwide (Gudaru et al.,
2020 ).
Since the awakening of the procedure 20 years ago, we witnessed
the enormous development in every aspect of AEEP. Therefore, we
believe that it is high time to summarise the advancement of the
technique so far. This Special Issue serves as a firm foundation by
compiling the achievements of researchers around the world and
shedding light on generalisation of the technique of AEEP.

Issue involves a comprehensive list of chapters and offers a con-
cise overview of AEEP in a systematic approach. Following the in-
troduction from the historical and anatomical perspective (Reddy,
Utley, \& Gilling, 2020; Oh \& Shitara, 2020), different techniques
of AEEP by different energy source are illustrated (Ryang, Ly, Tran,
Oh, \& Cho, 2020; de Figueiredo, Cracco, de Marins, \& Scoffone,
2020; Herrmann \& Wolters, 2020; Rijo \& Misrai, 2020). The surgical
outcomes, with emphasis on postoperative continence and sexual
functions, and complications of AEEP are summarised in the fol-
lowing chapters (Chen, Chung, Chu, Chen, \& Ho, 2020; Lee, Cho,
Juan, \& Teoh, 2020; Cheng, Li, \& Yu, 2020; Wei, Ke, Xu, \& Xue,
2020 ) before a debate on AEEP as the next gold standard treatment
for benign prostatic obstruction (Aho, Armitage, \& Kastner, 2020;
Wroclawski, Teles, \& Carneiro, 2020). Finally, this Special Issue is
rounded up by a discussion on surgical training (Teoh et al., 2020)
and a survey on AEEP from urologists worldwide (Gudaru et al.,
2020 ).
Since the awakening of the procedure 20 years ago, we witnessed
the enormous development in every aspect of AEEP. Therefore, we
believe that it is high time to summarise the advancement of the
technique so far. This Special Issue serves as a firm foundation by
compiling the achievements of researchers around the world and
shedding light on generalisation of the technique of AEEP.

Issue involves a comprehensive list of chapters and offers a con-
cise overview of AEEP in a systematic approach. Following the in-
troduction from the historical and anatomical perspective (Reddy,
Utley, \& Gilling, 2020; Oh \& Shitara, 2020), different techniques
of AEEP by different energy source are illustrated (Ryang, Ly, Tran,
Oh, \& Cho, 2020; de Figueiredo, Cracco, de Marins, \& Scoffone,
$2020 ;$ Herrmann \& Wolters, 2020; Rijo \& Misrai, 2020). The surgical
outcomes, with emphasis on postoperative continence and sexual
functions, and complications of AEEP are summarised in the fol-
lowing chapters (Chen, Chung, Chu, Chen, \& Ho, 2020; Lee, Cho,
Juan, \& Teoh, 2020; Cheng, Li, \& Yu, 2020; Wei, Ke, Xu, \& Xue,
2020 ) before a debate on AEEP as the next gold standard treatment
for benign prostatic obstruction (Aho, Armitage, \& Kastner, 2020;
Wroclawski, Teles, \& Carneiro, 2020). Finally, this Special Issue is
rounded up by a discussion on surgical training (Teoh et al., 2020)
and a survey on AEEP from urologists worldwide (Gudaru et al.,
2020 ).
Since the awakening of the procedure 20 years ago, we witnessed
the enormous development in every aspect of AEEP. Therefore, we
believe that it is high time to summarise the advancement of the
technique so far. This Special Issue serves as a firm foundation by
compiling the achievements of researchers around the world and
shedding light on generalisation of the technique of AEEP.

Issue involves a comprehensive list of chapters and offers a con-
cise overview of AEEP in a systematic approach. Following the in-
troduction from the historical and anatomical perspective (Reddy,
Utley, \& Gilling, 2020; Oh \& Shitara, 2020), different techniques
of AEEP by different energy source are illustrated (Ryang, Ly, Tran,
Oh, \& Cho, 2020; de Figueiredo, Cracco, de Marins, \& Scoffone,
$2020 ;$ Herrmann \& Wolters, 2020; Rijo \& Misrai, 2020). The surgical
outcomes, with emphasis on postoperative continence and sexual
functions, and complications of AEEP are summarised in the fol-
lowing chapters (Chen, Chung, Chu, Chen, \& Ho, 2020; Lee, Cho,
Juan, \& Teoh, 2020; Cheng, Li, \& Yu, 2020; Wei, Ke, Xu, \& Xue,
2020 ) before a debate on AEEP as the next gold standard treatment
for benign prostatic obstruction (Aho, Armitage, \& Kastner, 2020;
Wroclawski, Teles, \& Carneiro, 2020). Finally, this Special Issue is
rounded up by a discussion on surgical training (Teoh et al., 2020)
and a survey on AEEP from urologists worldwide (Gudaru et al.,
2020 ).
Since the awakening of the procedure 20 years ago, we witnessed
the enormous development in every aspect of AEEP. Therefore, we
believe that it is high time to summarise the advancement of the
technique so far. This Special Issue serves as a firm foundation by
compiling the achievements of researchers around the world and
shedding light on generalisation of the technique of AEEP.

\section{KEYWORDS}

enucleation, prostate

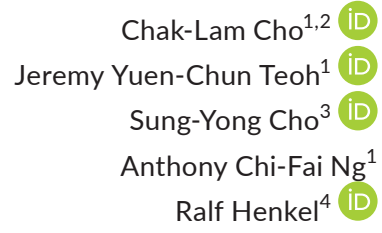


Correspondence

Chak-Lam Cho, Consultant in Urology, Department of Surgery, Union Hospital, 18 Fu Kin Street, Tai Wai, Shatin,

Hong Kong.

Email: chochaklam@yahoo.com.hk

\section{ORCID}

Chak-Lam Cho iD https://orcid.org/0000-0003-2520-6833

Jeremy Yuen-Chun Teoh iD https://orcid.

org/0000-0002-9361-2342

Sung-Yong Cho (iD https://orcid.org/0000-0001-9271-6951

Ralf Henkel iD https://orcid.org/0000-0003-1128-2982

\section{REFERENCES}

Aho, T., Armitage, J., \& Kastner, C. (2020). Anatomical endoscopic enucleation of the prostate: The next gold standard? Yes! Andrologia, 52, e13643. https://doi.org/10.1111/and.13643

Chen, C.-H., Chung, C.-H., Chu, H.-C., Chen, K.-C., \& Ho, C.-H. (2020). Surgical outcome of anatomical endoscopic enucleation of the prostate: A systemic review and meta-analysis. Andrologia, 52, e13612. https://doi.org/10.1111/and.13612

Cheng, B.- K.-C., Li, T.- C.-F., \& Yu, C.- H.-T. (2020). Sexual outcomes of endoscopic enucleation of prostate. Andrologia, 52, e13724. https:// doi.org/10.1111/and.13724

de Figueiredo, F. C. A., Cracco, C. M., de Marins, R. L., \& Scoffone, C. M. (2020). Holmium laser enucleation of the prostate: Problem-based evolution of the technique. Andrologia, 52, e13582. https://doi. org/10.1111/and.13582

Fraundorfer, M. R., \& Gilling, P. J. (1998). Holmium:YAG laser enucleation of the prostate combined with mechanical morcellation: Preliminary results. European Urology, 33(1), 69-72. https://doi. org $/ 10.1159 / 000019535$

Freyer, P. J. (1919). Total enucleation of the prostate. A further series of 550 cases of the operation. British Medical Journal, 1(3031), 121-120.2.

Gilling, P. J., Wilson, L. C., King, C. J., Westenberg, A. M., Frampton, C. M., \& Fraundorfer, M. R. (2012). Long-term results of a randomized trial comparing holmium laser enucleation of the prostate and transurethral resection of the prostate: Results at 7 years. BJU International, 109(3), 408-411. https://doi.org/10.1111/j.1464-410X.2011.10359.x

Gravas, S., Bach, T., Bachmann, A., Drake, M., Gacci, M., Gratzke, C., ...Tikkinen, K. A. O. (2016). EAU Guidelines on management of non-neurogenic male lower urinary tract symptoms (LUTS), incl. benign prostatic obstruction (BPO). https://uroweb.org/wp-conte
nt/uploads/EAU-Guidelines-Management-of-non-neurogenic-maleLUTS-2016.pdf

Gudaru, K., Gonzalez Padilla, D. A., Castellani, D.,Tortolero Blanco, L., Tanidir, Y., Ka Lun, L., ... Yuen-Chun Teoh, J. (2020). A global knowledge, attitudes and practices survey on anatomical endoscopic enucleation of prostate for benign prostatic hyperplasia among urologists. Andrologia, 52, e13717. https://doi.org/10.1111/and.13717

Herrmann, T. R. W., \& Wolters, M. (2020). Transurethral anatomical enucleation of the prostate with Tm:YAG support (ThuLEP): Evolution and variations of the technique. The inventors' perspective. Andrologia, 52, e13587. https://doi.org/10.1111/and.13587

Hiraoka, Y. (1983). A new method of prostatectomy, transurethral detachment and resection of benign prostatic hyperplasia. Nihon Ida Daigaku Zasshi, 50(6), 896-898. https://doi.org/10.1272/jnms1 923.50.896

Lee, H. Y., Cho, S. Y., Juan, Y.-S., \& Teoh, J.- Y.-C. (2020). How to optimise urinary continence in anatomical endoscopic enucleation of the prostate? Andrologia, 52, e13621. https://doi.org/10.1111/and.13621

Oh, S.-J., \& Shitara, T. (2020). Enucleation of the prostate: An anatomical perspective. Andrologia, 52, e13744. https://doi.org/10.1111/ and.13744

Rassweiler, J., Teber, D., Kuntz, R., \& Hofmann, R. (2006). Complications of transurethral resection of the prostate (TURP) - incidence, management and prevention. European Urology, 50(5), 969-980. https:// doi.org/10.1016/j.eururo.2005.12.042

Reddy, S. K., Utley, V., \& Gilling, P. J. (2020). The evolution of endoscopic prostate enucleation: A historical perspective. Andrologia, 52, e13673. https://doi.org/10.1111/and.13673

Rijo, E., \& Misrai, V. (2020). En bloc GreenLight laser enucleation of the prostate (GreenLEP): An in-depth look at the anatomical endoscopic enucleation of the prostate using a 532-nm lithium triborate laser. Andrologia, 52, e13729. https://doi.org/10.1111/and.13729

Ryang, S. H., Ly, T. H., Tran, A. V., Oh, S.-J., \& Cho, S. Y. (2020). Bipolar enucleation of the prostate-step by step. Andrologia, 52, e13631. https://doi.org/10.1111/and.13631

Teoh, J.- Y.-C., Cho, C.-L., Wei, Y., Isotani, S., Tiong, H.-Y., Ong, T.-A., ... the Asian Urological Surgery Training \& Education Group (2020). Surgical training for anatomical endoscopic enucleation of the prostate. Andrologia, 52, e13708. https://doi.org/10.1111/and.13708

Wei, Y., Ke, Z.-B., Xu, N., \& Xue, X.-Y. (2020). Complications of anatomical endoscopic enucleation of the prostate. Andrologia, 52, e13557. https://doi.org/10.1111/and.13557

Wroclawski, M. L., Teles, S. B., \& Carneiro, A. (2020). Anatomical endoscopic enucleation of the prostate: The next gold standard? No! (or not yet!). Andrologia, 52, e13707. https://doi.org/10.1111/and.13707 